\title{
Virtual Gas Sensor Array by Cyclic Optical Activation: Optimization of Activation Profile by Machine Learning
}

\author{
Gerald Njio ${ }^{1}$, Thorsten Wagner ${ }^{1}$ \\ 1 Paderborn University, Department of Chemistry, Warburger Str. 100, 33098 Paderborn, Germany, \\ thorsten.wagner@upb.de
}

\begin{abstract}
:
Optical activation of semiconducting metal oxide based gas sensors offers new ways of improving the performance of these cost-efficient and highly sensitive devices. Exposure to light in the UV/VIS range - as opposed to high temperature operation - is a low energy alternative to speed-up reaction kinetics and to balance adsorption-desorption equilibrium. Yet no study explicitly utilizes optical activation to compensate for the inherent lack in selectivity of the semiconducting sensors. In the following we present a new approach for generating a virtual gas sensor array by cyclic optical activation and a method to optimize activation profiles by utilizing supervised learning algorithms. The presentation is focused on nanostructured indium oxide $\left(\ln _{2} \mathrm{O}_{3}\right)$. However, the described method can be applied to various oxides used for semiconducting sensing as e.g. tin oxide or tungsten oxide. Different activation parameters like shape of the intensity curve, the intensity itself as well as the duration of the light exposure are evaluated by a neural network. Results are used to implement a signal stabilization algorithm for humidity and ozone sensing utilizing $\ln _{2} \mathrm{O}_{3}$.
\end{abstract}

Key words: optical activation, nanostructured metal oxide, semiconducting sensor, multi-signal generation, ozone

\section{Multi Signal Generation}

Different illumination profiles are already in use for photo-enhanced sensing, e.g. it was shown that continuous illumination by light in the UV/VIS range enhances the sensitivity of the nanostructured $\mathrm{In}_{2} \mathrm{O}_{3}$ sensor for $\mathrm{NO}_{2}[1]$ and $\mathrm{O}_{3}$ [2] and that cyclic operation allows for sensitivity increase even further [3]. In the following, however, cyclic activation will be utilized to generate multiple signals and enhance sensors selectivity and stabilize signals, respectively.

\section{Activation Profile Optimization}

In a first step a machine learning technique (artificial neural network, ANN) is applied to evaluate the sensing response of optically activated $\ln _{2} \mathrm{O}_{3}$-layers to variable relative humidity in air at room temperature. Figure 1 shows the probed activation profiles and the respective normalized sensor responses. Due to different effects, as e.g. photo-desorption, the response to illumination is delayed, i.e. during the presented cycles with a length of $10 \mathrm{~s}$ sensors do not reach stable resistance.

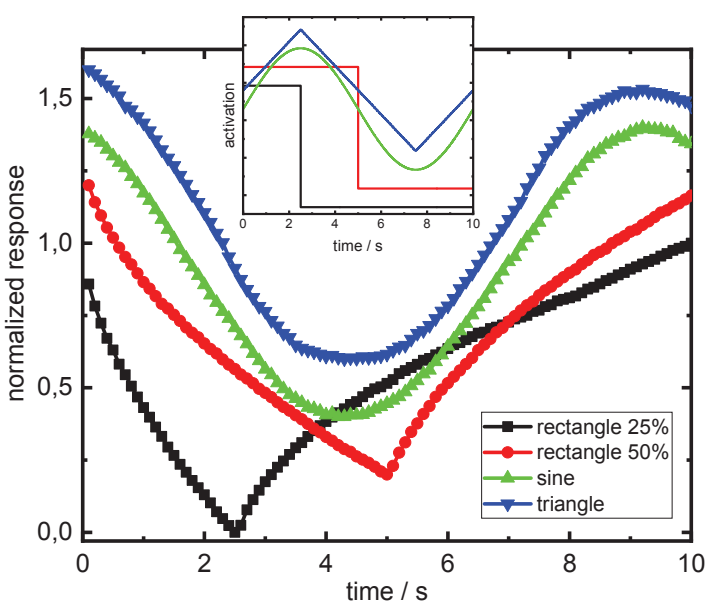

Fig. 1. Normalized sensor response of an $\operatorname{In}_{2} \mathrm{O}_{3}$ layer for different intensity profiles of the cyclic optical activation (inset). (room temperature, illumination wavelength $460 \mathrm{~nm}, 20 \%$ rel. humidity in synth. Air)

In addition it can be observed, that the curvature in the case of a rectangular shaped intensity profile strongly depends on the illumination time $(25 \%$ or $50 \%$ of total cycle time, respectively).To evaluate the information content of the response, a data set of 500 cycles with known humidity (7 target classes) 
was utilized to train an ANN (4-layer perceptron). As can be seen in figure 2, high recognition efficiency can be achieved by this method.

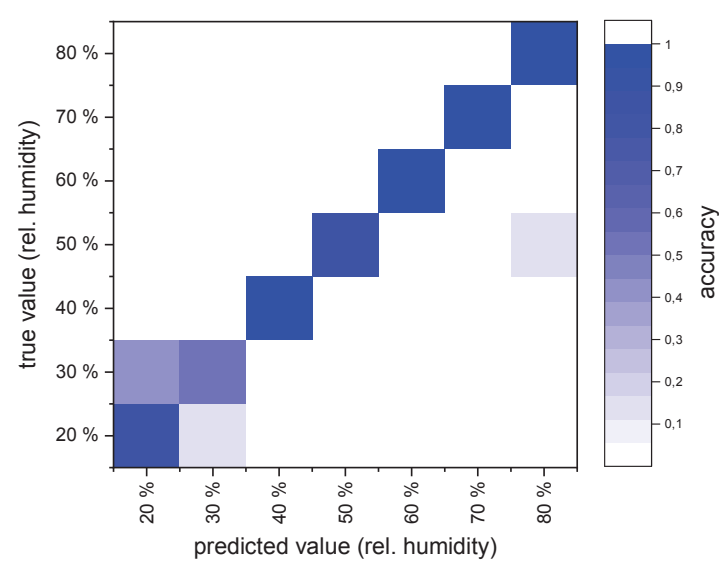

Fig. 2. Confusion matrix of a test of an optimized multilayer perceptron with an overall efficiency $86 \%$.

For the cycles presented in figure 1, the highest efficiency according to the ANN is attained by the rectangular intensity profile with $2.5 \mathrm{~s}$ illumination time. The recognition rate for the other cycle types drops significantly to $34 \%$ and $26 \%$ for the sinusoidal and the triangular shaped profile, respectively. In addition to the optimization of the activation profile, detailed analysis of the single neurons and the input weights allows identification of the most significant data points in the response cycle. These cycle points are utilized to develop more simple evaluation algorithms.

\section{Signal Stabilization and Reaction Time}

The above described analysis gives insight into relevant time constants of the underlying chemical processes. Based on the response time caused by desorption of chemisorbed surface species during illumination, it is possible to determine an indicator for the relative surface coverage of the semiconducting layer. Two application examples, showing signal stabilization for humidity measurements and an improved regeneration characteristics for room temperature ozone sensing, are presented. In the latter case signal recovery can be achieved in the range of 10 seconds compared to 30 minutes by proper multi-signal evaluation. With this system operation even at room temperature seems to be possible for certain analyte-material combinations.

\section{References}

[1] T. Wagner, C.-D. Kohl, C. Malagù, N. Donato, M. Latino, G. Neri and M. Tiemann, Sensors and Actuators B: Chemical 187, 488-494 (2013); doi: 10.1016/j.snb.2013.02.025

[2] T. Wagner, J. Hennemann, C.-D. Kohl and M. Tiemann, Thin Solid Films, 520 918-921 (2011); doi: 10.1016/j.tsf.2011.04.181

[3] Ch.Y. Wang, R.W. Becker, T. Passow, W. Pletschen, K. Köhler, V. Cimalla, O. Ambacher, Sensors and Actuators B: Chemical 152, 235-240 (2011); doi: 10.1016/j.snb.2010.12.014 